\title{
Entrenamiento de la visualización espacial mediante ejercicios informatizados de dibujo técnico
}

\author{
Entrenamiento de la visualización especial
}

\author{
Gerardo Prieto \\ Angela D. Velasco
}

\begin{abstract}
Resumen
En este trabajo se describe la construcción, la aplicación y los resultados obtenidos con una batería de ejercicios informatizados para entrenar la Visualización Espacial de estudiantes de Ingeniería. La batería contiene cuatro ejercicios basados en tareas muy comunes en la enseñanza básica del Dibujo Técnico. Cada ejercicio se compone de 18 ítems con cuatro opciones de respuestas de las que una es correcta. Tras la respuesta a cada ítem, el alumno recibe feed-back inmediato y con demostración de la precisión de su respuesta. El formato de respuesta de los ejercicios se denomina "Responder hasta acertar", dado que si la respuesta fue incorrecta, el alumno debe buscar de nuevo la solución. Al terminar la prueba, el alumno recibe información sobre la puntuación obtenida. Para evaluar la influencia del entrenamiento en la Visualización Espacial, se administraron tests de esa aptitud, escalados en la misma métrica, al comienzo y al final del curso de Dibujo Técnico. Las figuras de los ejercicios y los tests fueron construidas con AutoCad y la programación fue realizada con Revolution Studio 2. Se utilizaron varios modelos para obtener las medidas: Partial Credit Model (Masters, 1982) y Rasch Model (Rasch, 1960). Se observó que, en promedio, la mejora de los alumnos en Visualización Espacial fue moderada.
\end{abstract}

Palabras clave: Visualización espacial, Dibujo técnico, Feedback, Educación.

\section{Training spatial visualization using computer exercises of technical drawing}

\begin{abstract}
This work describes the construction, application and results obtained with a battery of computerized exercises for training Spatial Visualization in engineering students. The battery contains four exercises based on very common tasks in the basic teaching of Technical Drawing. Each exercise is composed of 18 items with four options to answer from, only one of which is correct. After answering each item, the student receives immediate feedback of whether the answer is right or wrong. The response format of the exercises is called "Answer-Until-Correct," because if the answer the student gives is incorrect, he or she must keep on seeking the right answer. When the test is over, the student receives information on the score obtained. To assess the effectiveness of the training in Spatial Visualization, tests in this ability, scaled in the same metrics, were administered at the beginning and end of a Technical Drawing course. The figures of the exercises and tests were constructed with AutoCad and programming was carried out with Revolution Studio 2. Two models were used to obtain the measures: the Partial Credit Model [1] and the Rasch Model [2]. It was observed that, on average, the students' improvement in Spatial Visualization was moderate.
\end{abstract}

Key words: Visuospatial ability, Technical drawing, Feedback, Education.

\section{Treinamento da visualização espacial mediante exercícios informatizados de desenho técnico}

\begin{abstract}
Resumo
Neste trabalho descreve-se a construção, a aplicação e os resultados obtidos numa bateria de exercícios informatizados para treinamento da visualização espacial de estudantes de Engenharia. A bateria contém quatro exercícios baseados em tarefas muito comuns do ensino fundamental de desenho técnico. Cada exercício é composto por 18 itens com quatro opções de respostas das quais apenas uma é correta. Após responder a cada item o aluno recebe um feed-back imediato, com a demonstração da precisão da sua resposta. O formato de resposta dos exercícios denomina-se "Responder até acertar", uma vez que se a resposta é incorreta, o aluno recebe a informação da pontuação obtida. Para avaliar a influencia do treinamento na visualização espacial foram administrados testes dessa aptidão no começo e no final do curso de desenho técnico. As figuras dos exercícios e os testes foram construídas com AutoCad e a programação foi realizada com Revolution Studio 2. Utilizaram-se vários modelos para obter as medidas: Partial Credit Model (Masters, 1982) e Rasch Model (Rasch, 1960). Observou-se que os alunos apresentaram uma melhora moderada em visualização espacial.

Palavras-chave: Visualização espacial, Desenho técnico, Feedback, Educação.
\end{abstract}




\section{Introducción}

En los más importantes estudios factoriales con tests espaciales (Carroll, 1993; Lohman, 1988; McGee, 1979; Michael , Guilford, Fruchter \& Zimmerman, 1957), se ha concluido que la Visualización, definida como la aptitud para manipular, rotar, torcer o invertir las imágenes de los objetos es el factor más importante de las aptitudes espaciales. Desde una perspectiva aplicada, los tests de Visualización se han mostrado como predictores eficaces del éxito académico y profesional de arquitectos, cirujanos, diseñadores gráficos, ingenieros y pilotos (Gibbons, Baker, \& Skinner, 1986; Hegarty \& Simms, 1994; Hsi, Linn, \& Bell, 1997; Lohman, 1994; Prieto \& Velasco, 2002).

La susceptibilidad de las aptitudes espaciales al entrenamiento es uno de los temas incluidos en los programas de investigación. Este interés por el entrenamiento radica en sus consecuencias en el éxito académico y laboral: la mejora de las aptitudes beneficiaría el aprendizaje y el rendimiento en profesiones técnicas como las mencionadas anteriormente (Newcombe, Mathason \& Terlecki, 2002).

Baenninger y Newcombe (1989) publicaron un metaanálisis de los estudios acerca de la entrenabilidad de las aptitudes espaciales. Los estudios analizados fueron clasificados en varias categorías en función del contenido del entrenamiento (específico, general o indirecto) y de su duración (larga, media y corta). El término "específico" se refiere a la preparación en un único test espacial y el término "general" al entrenamiento en una variedad de tests espaciales. Los entrenamientos denominados "indirectos" incluyen actividades asociadas con la aptitud espacial, pero que no están dirigidas a instruir en tests espaciales específicos. En lo que se refiere a la duración, consideraron "cortos" los entrenamientos inferiores a tres semanas, "medios" los que duraron más de tres semanas y menos de un semestre, y "largos" los que emplearon un semestre o más. Estos últimos suelen coincidir con la instrucción formal en materias (por ejemplo, Dibujo Técnico) incluidas en currícula técnicos, como ingeniería y arquitectura.

De los estudios citados se pueden extraer algunas conclusiones relevantes. En primer lugar, parece claro que el entrenamiento incrementa el rendimiento en tests espaciales. En segundo lugar, es evidente que los mayores beneficios se suelen obtener cuando en el entrenamiento se utilizan materiales muy semejantes a los ítems de los tests espaciales empleados para medir el cambio aptitudinal. A nuestro juicio, con el fin de evitar efectos meramente artificiales y extraer conclusiones generalizables, se han de preferir entrenamientos indirectos con tareas distintas a las de los tests.
Finalmente, se manifiesta una escasez de estudios sobre entrenamientos de larga duración, sobre todo con la aptitud de Visualización.

Por ello, el objetivo de este trabajo es evaluar el efecto en la Visualización de un entrenamiento indirecto de larga duración, consistente en un curso semestral de Dibujo Técnico auxiliado por ejercicios informatizados.

En este trabajo se describen las características de una batería de ejercicios informatizados construida para entrenar indirectamente la Visualización mediante tareas que son comunes en un curso de Dibujo Técnico, y que fueron introducidas durante un curso semestral de esa materia dirigido a alumnos del primer curso de Ingeniería. Además de describir las características de la batería, se analiza la mejora de la Visualización Espacial tras el seguimiento del curso de Dibujo Técnico.

\section{Los Ejercicios}

La batería fue construida con el programa Revolution Studio 2, de Runtime Revolution Limited (https://secure.runrev.com) y las figuras tridimensionales con AutoCad. Se compone, por el momento, de cuatro ejercicios basados en tareas comunes a la enseñanza básica del Dibujo Técnico. Todas las representaciones en perspectiva de los objetos están en isométricas. Los cuatro ejercicios tienen como introducción un breve resumen del contenido de la tarea que está ilustrada con textos breves y gráficos animados. Cada ejercicio se compone de 18 ítems con cuatro opciones de respuestas de las que una es correcta. Tras la respuesta a cada ítem, el alumno recibe feed-back inmediato de la precisión de su respuesta (acierto/error). Asimismo, recibe una demostración animada de la corrección o incorrección. Se facilita feedback aún en el caso de que la respuesta haya sido correcta para que, si el alumno hubiese acertado por adivinación, verifique la solución. Este formato de respuesta se denomina "Responder hasta acertar", dado que si la respuesta fue incorrecta, el alumno debe buscar de nuevo la solución (Wilcox, 1981). El programa almacena las respuestas a los 18 ítems y calcula una puntuación que refleja la competencia del alumno en el ejercicio. Al terminar la prueba, el alumno recibe información sobre la puntuación obtenida.

El primer ejercicio se basa en una tarea de rotación mental de figuras tridimensionales. El alumno debe reconocer la posición de una arista o una cara de un objeto rotado en el espacio (Ver la Figura 1).

En los ítems del segundo ejercicio, se le pide al alumno que elija la opción en la que aparecen correctamente las principales vistas ortogonales de un objeto (Ver la Figura 2). 


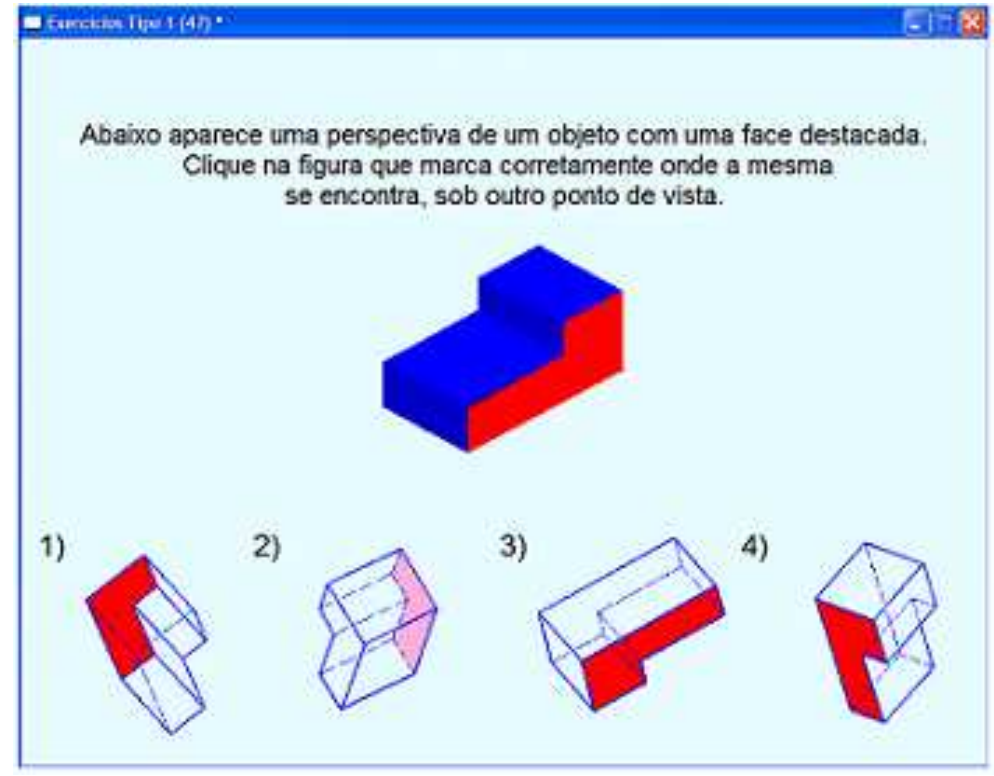

Figura 1. Ejemplo de un ítem del Ejercicio 1. Se pide al alumno que marque la opción en la cual aparece marcada en rojo la misma cara que en la de la figura de arriba.

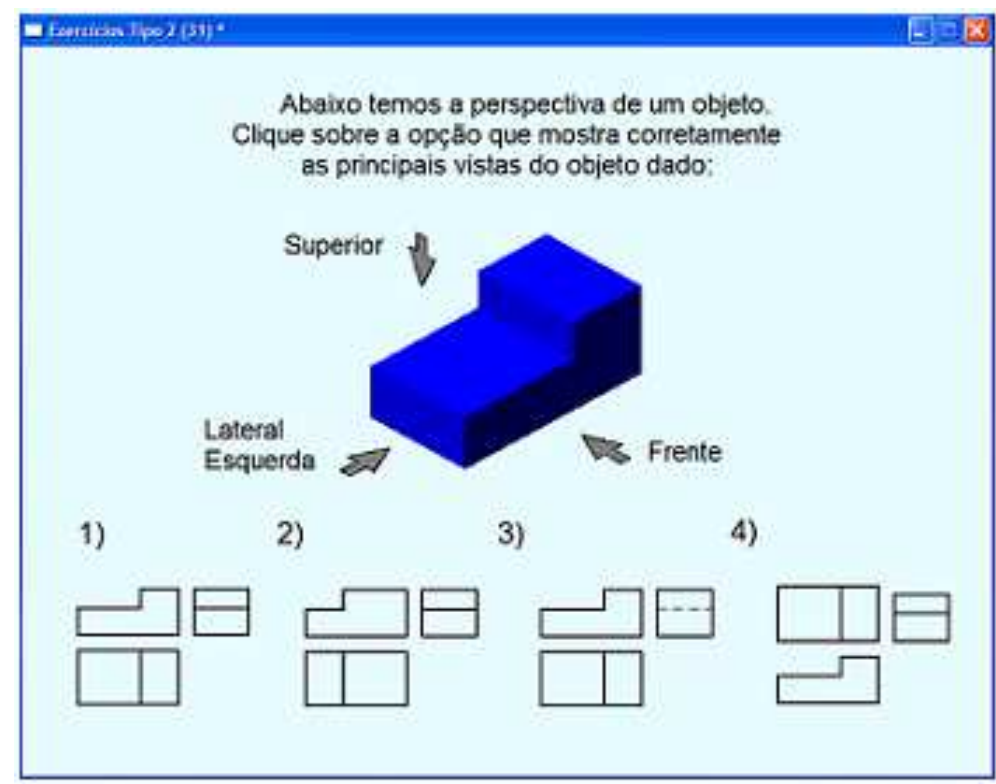

Figura 2. Ejemplo de un ítem del Ejercicio 2. Se pide al alumno que marque la opción que muestra las principales vistas de la figura de arriba.

En los ítems del tercer ejercicio, se presentan las vistas ortogonales principales de un objeto. El alumno debe identificar el objeto al que corresponden las vistas (Ver Figura 3). 


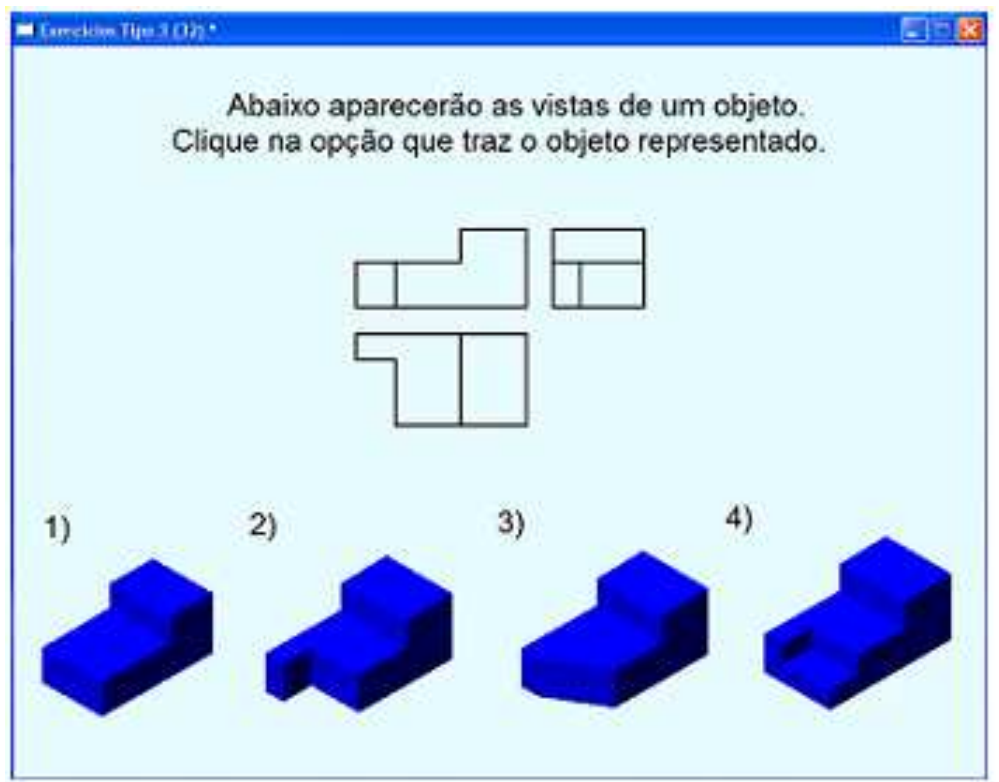

Figura 3. Ejemplo de un ítem del Ejercicio 3. Se pide al alumno que marque la opción correspondiente a las vistas que aparecen en la parte superior.

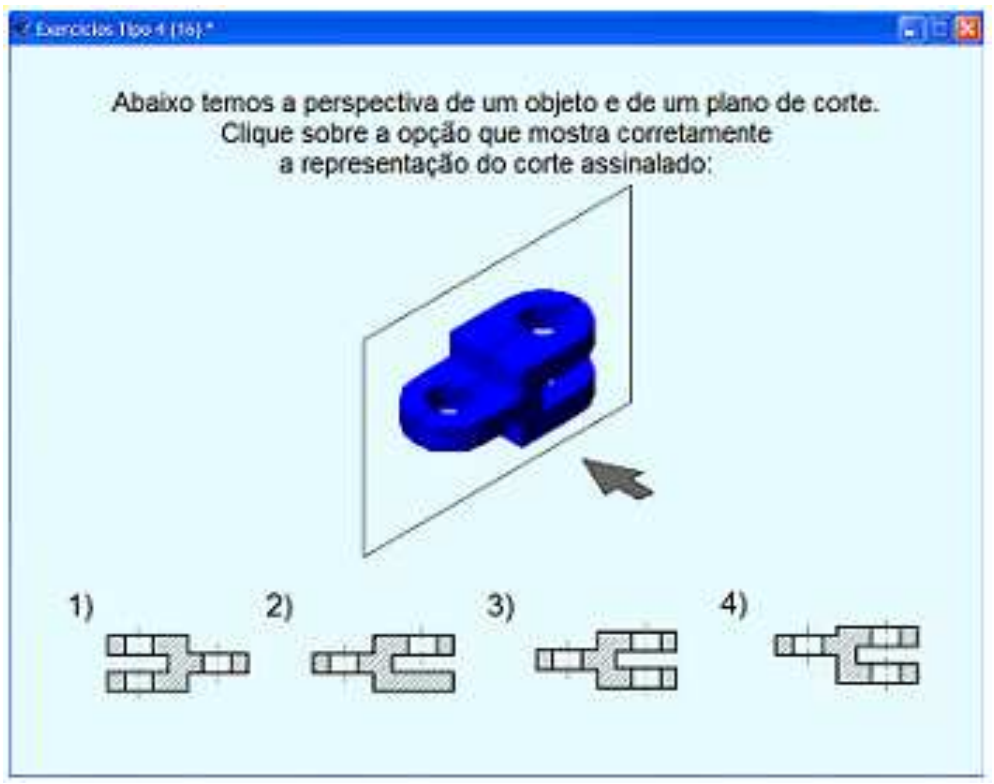

Figura 4. Ejemplo de un ítem del Ejercicio 4. Se pide al alumno que marque la opción que corresponde al corte en el plano de la figura superior.

El cuarto ejercicio se basa en una tarea de reconocimiento de cortes (comprensión de las partes internas de un objeto).

Para elaborar las opciones distractoras de los ejercicios 2, 3 y 4, se tomaron en consideración los errores más frecuentes de los alumnos al realizar ese tipo de ejercicios con formato de papel y lápiz en cursos anteriores (por ejemplo, errores en la proporción de las figuras, en la posición de las vistas, en los tipos de línea, ausencia de aristas, etc).
Como se ha indicado anteriormente, los ítems son de elección múltiple y están integrados por cuatro opciones de respuesta de las que una es correcta. Después de cada respuesta el alumno recibe información sobre su corrección o incorrección y un feed-back de demostración para que realice una verificación y mejore su aprendizaje. $\mathrm{Si}$ la respuesta fue incorrecta, se le obliga a que busque de nuevo la solución. Es decir, no es posible pasar al ítem siguiente hasta que la respuesta no sea correcta (Véase la Figura 5). 


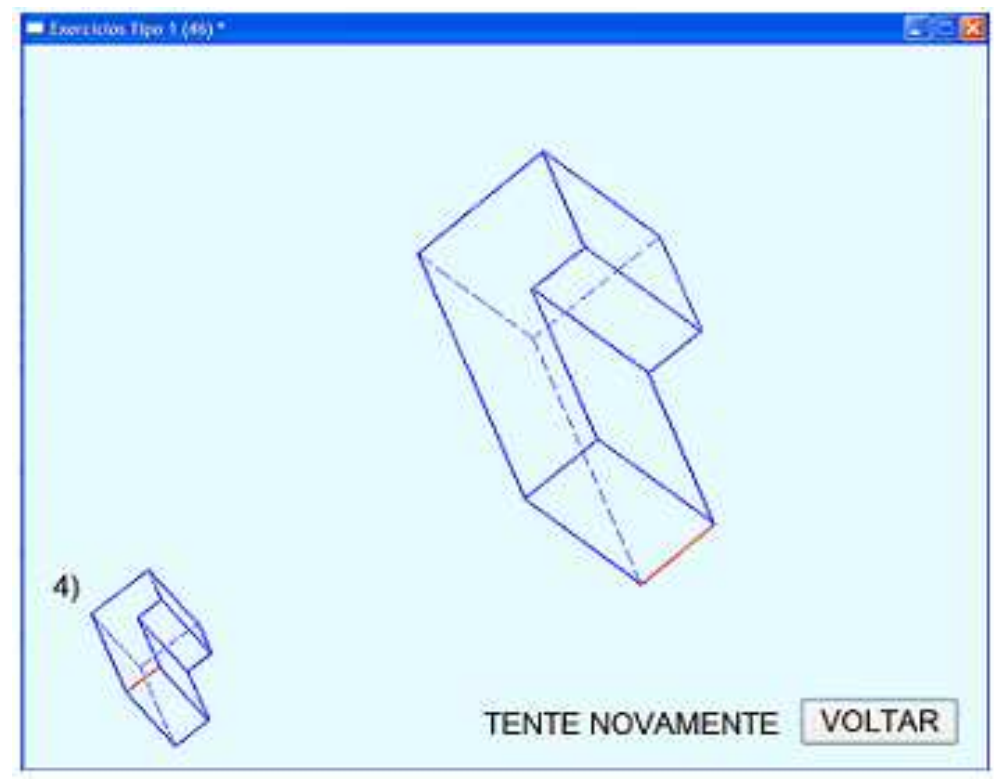

Figura 5. Después de observar que su respuesta es errónea (figura menor), se pide al alumno que elija otra opción.

\section{Método}

\section{Muestra}

Los participantes fueron 104 alumnos del primer año de Ingeniería en la UNESP (Campus de Guaratinguetá). La media y desviación típica de la edad eran de 19,25 y 1,89 respectivamente. El $76 \%$ de los participantes eran hombres.

\section{Instrumentos}

1 Se administraron dos tests informatizados de Visualización (TVZ2006-A y TVZ2006-B), cuyos ítems se basaban en una tarea de Desarrollo de Superficies (Figura 6). Cada test se componía de 20 ítems con 9 opciones de respuesta de las que sólo una era correcta (Prieto \& Velasco, 2004). Los alumnos dispusieron de un tiempo máximo de 30 minutos para contestar a los ítems de cada test. Para decrementar el efecto de la memorización de los ítems y el efecto techo en el postest, se incluyeron dos tests de diferente nivel de dificultad. El test TVZ2006-A era más fácil que el test TVZ2006-B. Posteriormente, las puntuaciones (logits) de ambas pruebas fueron calibradas en la misma métrica mediante cuatro ítems de anclaje.

2 Los cuatro ejercicios de entrenamiento descritos anteriormente.

\section{Procedimiento}

El test TVZ2006-A fue aplicado al comienzo del curso de Dibujo Técnico, y el test TVZ2006-B al final. Entre ambas administraciones transcurrieron cinco meses. El curso de Dibujo Técnico es un curso teórico-práctico orientado a la representación gráfica de objetos. El propósito del curso es brindar al estudiante los conocimientos básicos de representación de objetos y el aprendizaje de un software para la elaboración de dibujos y planos. Contenidos del curso: Editor de dibujo de AutoCAD en el entorno Windows; Sistemas de coordenadas; Construcciones geométricas; Geometría aplicada; Capas; Acotado; Dibujos isométricos; Dibujo de objetos en 3D; Visualización de sólidos y Proyecciones múltiples o diédricas. Los cuatro ejercicios informatizados se incluyeron a lo largo del curso con una separación aproximada de un mes entre cada uno de ellos. 


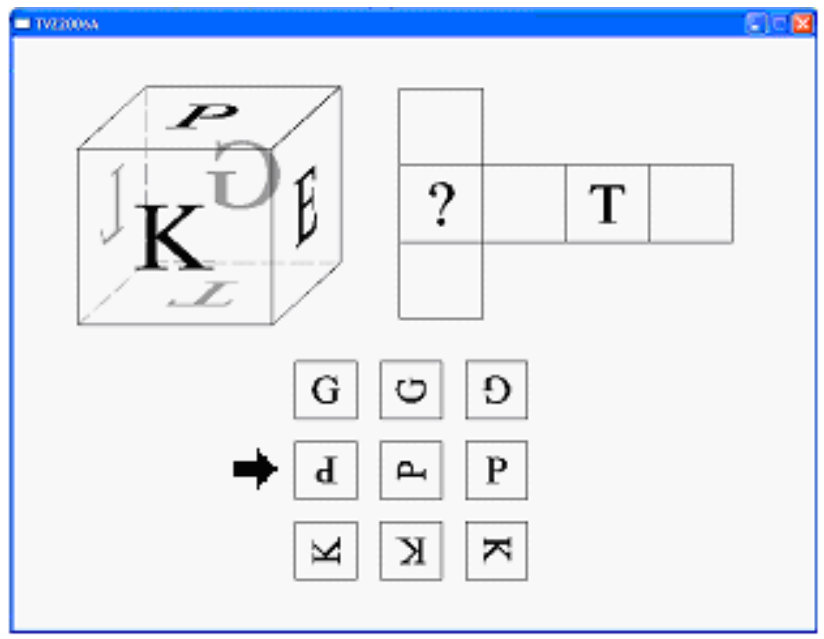

Figure 6. Ejemplo de un ítem de los tests de Vz. La opción correcta está indicada con una flecha.

\section{Modelos de medida}

Se utilizaron dos modelos muy conocidos de la Teoría de Respuesta al Ítem. El modelo de Rasch (1960) y el Modelo de Crédito Parcial (Masters, 1982). Se empleó el modelo de Rasch (1960) para obtener las medidas pre y post de la Visualización. La elección de este modelo se justifica por sus excelentes propiedades para medir el cambio (Embretson, 1987; Wolfe \& Chiu, 1999): medición de intervalo, estimación de errores estándar de medida específicos a lo largo de la variable y facilidad para equiparar en la misma métrica tests de distinta dificultad. El modelo de Rasch para respuestas dicotómicas (acierto/error) especifica que la probabilidad $(P)$ de que una persona resuelva correctamente un ítem, es una función de la diferencia aptitudinal entre la persona $(\mathrm{Bn})$ y el ítem (Di): loge (Pni / (1 - Pni )) = Bn-Di (Rasch, 1960).

Para analizar los datos de los ejercicios se empleó el modelo de Crédito Parcial, una extensión del modelo de Rasch para ítems politómicos, que indica que la probabilidad de la respuesta al ítem depende del nivel aptitudinal del sujeto $(\mathrm{Bn})$, de la dificultad del ítem (Di) y de la posición de la categoría de respuesta en el continuo medido (Fij): loge $($ Pnij/Pni(j-1) $)=$ Bn-Di-Fij. Específicamente,

Pnij = la probabilidad de que la respuesta del sujeto pertenezca a la categoría j (por ejemplo, acertar en el primer intento).

Pni(j-1) = la probabilidad de que la respuesta pertenezca a alguna de las categorías inferiores a $\mathrm{j}$ (acertar al segundo, tercero o cuarto intento).

$\mathrm{Fij}=$ el punto de transición (paso) en el continuo medido entre la categoría j y la categoría inferior.

Los aciertos a los ítems de los ejercicios recibieron distinto peso en función del orden en el que se produjeron: en el primer intento (4), en el segundo (2), en el tercero (1) o en el cuarto (0). En los estudios previos con el formato "Responder hasta acertar" se muestra que esta es la ponderación más efectiva (Dodd \& Koch, 1987; Hirose, 2000; Menéndez, 2008). Es decir, resolver el ítem correctamente en el primer intento indica mucho más nivel de competencia que en los intentos posteriores.

Para analizar los datos se empleó el programa WINSTEPS 3.64 (Linacre, 2007).

\section{Resultados}

En la Tabla 1 se muestran los estadísticos descriptivos del rendimiento en los ejercicios administrados.

Se observa que el rendimiento de los alumnos en los cuatro ejercicios es muy alto: las medias de los aciertos están muy cercanas a la máxima puntuación posible (72), y las medias de las puntuaciones Rasch (logits) son muy superiores a 0 (por convención, la dificultad media de los ítems). No obstante, aparece una variabilidad moderada en su ejecución. Aunque las altas puntuaciones en los ejercicios pudiesen atribuirse a la alta competencia de los alumnos, nos inclinamos más a considerar que la baja dificultad de los ítems es la principal causa del elevado rendimiento de los alumnos.

Excepto en el ejercicio 4, la fiabilidad o consistencia interna (Cronbach alpha) de las puntuaciones de los ejercicios es aceptable. 
Tabla 1. Estadísticos descriptivos de los ejercicios y los tests

\begin{tabular}{lcccccc}
\hline Estadístico & E1 & E2 & E3 & E4 & VZ-Pretest & VZ-Postest \\
\hline Media X & 63,5 & 64,4 & 67,0 & 66,6 & 12,5 & 12,1 \\
DT X & 7,0 & 6,20 & 5,40 & 4,0 & 4,6 & 5,3 \\
Media B & 1,39 & 1,49 & 1,71 & 1,51 & 0,77 & 1,52 \\
DT B & 0,74 & 0,66 & 0,68 & 0,60 & 1,39 & 1,80 \\
Alpha & 0,72 & 0,69 & 0,70 & 0,42 & 0,83 & 0,88 \\
R X-DT & $0,41^{*}$ & $0,37^{*}$ & 0,16 & $0,34^{*}$ & $0,27^{*}$ & 0,16 \\
R B-DT & $0,37^{*}$ & $0,46^{*}$ & 0,20 & 0,22 & $0,30^{*}$ & 0,18 \\
\hline
\end{tabular}

Media $\mathrm{X}=$ Media de las puntuaciones clásicas de las personas

DT $\mathrm{X}=$ Desviación típica de $\mathrm{X}$

Media B = Media de los parámetros de las personas (logits)

DT B = Desviación típica

Alpha $=$ Coeficiente alpha de Cronbach

R X-DT $=$ Correlación de X con la calificación en Dibujo Técnico

$\mathrm{R}$ B-DT= Correlación de B con la calificación en Dibujo Técnico

$* p<0,05$

En la Tabla 1 aparecen también los estadísticos de las puntuaciones en los tests de Visualización empleados en el pre y postest. Los alumnos presentan una gran variabilidad en los tests de aptitud. Es de destacar que la fiabilidad de las puntuaciones es muy alta, y mayor que la que se observa en los ejercicios de entrenamiento. La mejor fiabilidad de los tests se debe a que la dificultad de sus ítems es más adecuada para el nivel de aptitud de los alumnos.

Dado que las puntuaciones Rasch (B) del pretest y del postest de Visualización están en la misma escala, se puede observar que se ha producido una mejora de los alumnos tras el curso de Dibujo Técnico auxiliado por los ejercicios informatizados. La diferencia entre las medias del postest y el pretest $(0,75)$ es estadísticamente significativa (t de Student $=4,6 ; p=0,00$ ). Para valorar la magnitud de la mejora, se ha cuantificado el tamaño del efecto mediante el estadístico d de Cohen (1988), que expresa en desviaciones típicas la diferencia entre ambas medias. En este caso, el tamaño del efecto es medio $(\mathrm{d}=$ 0,56 ). No se aprecian diferencias significativas entre la mejora de los alumnos (media $=0,73 ; \mathrm{SD}=1,4$ ) y de las alumnas (media $=0,84 ; S D=1,0): t$ de Student $=-0,27 ; p=$ 0,14 .

En la Tabla 1 aparecen las correlaciones de las puntuaciones de los tests de aptitud y de los ejercicios con las calificaciones finales en la asignatura de Dibujo Técnico. Pese a la escasa variabilidad de las puntuaciones en los ejercicios, la mayoría de las correlaciones son moderadas y estadísticamente significativas. Estos datos ponen de manifiesto que el rendimiento en los ejercicios es un predictor del éxito en el examen final. Asimismo, se replican los resultados de los estudios en los que se concluye que la aptitud de Visualización está asociada con el aprendizaje del Dibujo Técnico (Halpern \& Collaer, 2005;

Hegarty \& Simms, 1994; Hsi \& cols., 1997; Lohman, 1994; Prieto \& Velasco, 2002).

\section{Conclusiones}

De este trabajo se pueden extraer dos conclusiones fundamentales.

En primer lugar, se ha presentado, una batería de ejercicios informatizados para contribuir al aprendizaje de los alumnos de ingeniería en la materia de Dibujo Técnico.

Las tareas de esta batería inciden en facilitar la representación mental de la estructura interna o las transformaciones de objetos tridimensionales. Se han concebido los ejercicios como un medio de entrenamiento. En consecuencia, se facilita un feed-back tras la respuesta del alumno y una demostración animada para que se comprenda la causa de la corrección o incorrección de la respuesta. En el caso de que la respuesta fuese incorrecta, se obliga al alumno a que busque de nuevo la solución. Este formato de respuesta, denominado responder hasta acertar, es considerado muy útil en la enseñanza asistida por la computadora. Las entrevistas informales llevadas a cabo a un pequeño grupo de alumnos pusieron de relieve que, tanto el contenido de los ejercicios como este formato de respuesta, resultaron muy atractivos y motivadores.

Adicionalmente, los ejercicios facilitan una puntuación para cada alumno que evalúa su competencia en el ejercicio. Las puntuaciones fueron obtenidas mediante el modelo de Crédito Parcial. Estas puntuaciones presentan una asociación moderada con las calificaciones finales en Dibujo Técnico.

En segundo lugar, se han obtenido evidencias de que la aptitud de Visualización, un clásico mediador del éxito en profesiones como la Ingeniería, la Arquitectura y el Diseño Gráfico, es modificable mediante entrenamientos 
indirectos de larga duración. En este trabajo, la mejora producida en alumnos de alto nivel aptitudinal como los de ingeniería, es de tipo medio, de acuerdo con la taxonomía de Cohen (1988). Este dato concuerda con los obtenidos por nosotros en estudios anteriores (Prieto \& cols., 2004, 2008).

Suponemos que se podría incrementar el tamaño del cambio, introduciendo más ejercicios y de mayor dificultad que los empleados en este estudio.

Finalmente, pese a la influencia de la restricción del rango en la magnitud de las correlaciones, se replica la asociación de la aptitud de Visualización con el rendimiento en el aprendizaje del Dibujo Técnico. Por ello, el uso de test de Visualización al inicio del curso permitiría identificar a los alumnos con probables problemas de aprendizaje, con el fin de prestarles una especial atención en el proceso de enseñanza.

\section{Referencias}

Baenninger, M., \& Newcombe, N. (1989). The role of experience in spatial test performance: A meta-analysis. Sex Roles, 20, 327-343.

Carroll, J. (1993). Human cognitive ability: A survey of factoranalytical studies. New York: Cambridge University Press.

Cohen, J. (1988). Statistical power analysis for the behavioral sciences. Hillsdale, NJ: Erlbaum.

Dodd, B. G., \& Koch, W. R. (1987). Effects of variations in item stepvalues on item and test information in the partial credit model. Applied Psychological Measurement, 11(4), 371-384.

Embretson, S. E. (1987). Improving the Measurement of Spatial Aptitude by Dynamic Testing. Intelligence, 11, 333-358.

Gibbons, R., Baker, R., \& Skinner, D. (1986). Field articulation testing: A predictor of technical skills in surgical residents. Journal of Surgical Research, 41, 53-47.

Halpern, D. F., \& Collaer, M. L. (2005). Sex Differences in Visuospatial Abilities: More Than Meets the Eye. En P. Shah \& A. Miyake (Eds.), The Cambridge Handbook of Visuospatial Thinking (pp-170-212). Cambridge: Cambridge University Press.

Hegarty, M., \& Simms, V. K. (1994). Individual differences in mental animation during mechanical reasoning. Memory and Cognition 22, 411-430.

Hirose, I. (2000). Answer-Until-Correct Item Response Model with Restricted Number of Responses. Japan Journal of Educational Technology 24, 53-62.

Hsi, S., Linn, M. C., \& Bell, J. E. (1997). The role of spatial reasoning in enginnering and design of spatial instructions. Journal of Engineering Education, 86, 151-158.
Linacre, J.M. (2007). A User's Guide to WINSTEPS \& MINISTEPS: Rasch Model Computers Programs. Chicago IL: Winsteps.com.

Lohman, D. F. (1994). Spatial Ability. En R. Sternberg (Ed.), Encyclopedia of Human Intelligence (pp. 1000-1007). New York: McMillan.

Lohman, D. F. (1988). Spatial abilities as traits, processes, and knowledge. En R. J. Sternberg (Ed.), Advances in the psychology of human intelligence (pp. 181-248). Hillsdale, NJ: Lawrence Erlbaum.

Masters, G. N. (1982). A Rasch model for partial credit scoring. Psychometrika, 47, 149-174.

McGee, M. G. (1979). Human spatial abilities: Psychometric studies and environmental, genetic, hormonal, and neurological influences. Psychological Bulletin, 86, 889-918.

Menéndez, L. F. (2008). Modelos psicométricos para el análisis de ítems aplicados con el formato responder hasta acertar. Tesis Doctoral, Universidad de Oviedo, España.

Michael, W. B., Guilford, J. P., Fruchter, B., \& Zimmerman, W. S. (1957). The description of spatial-visualization abilities. Educational and Psychological Measurement, 17, 185-199.

Newcombe, N. S., Mathason, L., \& Terlecki, M. (2002). Maximization of Spatial Competence: More Important than Finding the Cause of Sex Differences. En A. McGillicudy-De Lisi \& R. De Lisi (Eds.), Biology, Society, and Behavior: The development of sex differences in cognition (pp. 183-206). Wesport, CT: Ablex.

Prieto, G., \& Velasco, A. D. (2002). Predicting academic success of engineering students in technical drawing from visualization test scores. Journal for Geometry and Graphics, 6, 99-109.

Prieto, G., \& Velasco, A. D. (2004). Training Visualization Ability by Technical Drawing. Journal for Geometry and Graphics, 8, 107115.

Prieto, G., Velasco, A. D., Arias-Barahona, R., Anido, M., Núñez, A. \& Có, P. (2008). ¿Mejora la visualización espacial con el aprendizaje del dibujo técnico? Revista Mexicana de Psicología, 25, 175-182.

Rasch, G. (1960). Probabilistic models for some intelligence and attainment tests. Chicago: University of Chicago Press.

Wilcox, R. (1981). Solving Measurement Problems with an answerUntil-Correct Scoring Procedure. Applied Psychological Measurement, 7, 67-74.

Wolfe, E. W., \& Chiu, C. W. T. (1999). Measuring Pretest-Posttest Change with a Rasch Rating Scale Model. Journal of Outcome Measurement, 3, 134-161. 
Recebido em: 28/11/2008

Revisado em 30/11/2008

Aprovado em 02/11/2008

\section{Sobre os autores}

Gerardo Prieto (gprieto@usal.es) - Universidad de Salamanca - España

Angela D. Velasco (http://www.feg.unesp.br) - professor assistente doutor da Universidade Estadual Paulista Júlio de Mesquita Filho.

Dirección para la correspondencia:

Gerardo Prieto. Departamento de Psicología Básica, Psicobiología y Metodología. Avda. de la Merced 109-131. 37005 Salamanca - España. E-mail: gprieto@usal.es.

\section{Nota dos autores}

Agradecimiento:

Este trabajo fue financiado por la Dirección General de Investigación del Ministerio de Educación y Ciencia (España). Referencia del proyecto: SEJ2007-61118. 
